\title{
@creative
commons
}

ISSN 2590-9770

The Art of Discrete and Applied Mathematics 6 (2023) \#P2.07

https://doi.org/10.26493/2590-9770.1484.40e

(Also available at http://adam-journal.eu)

\section{Mycielskian of graphs with small game domination number}

\author{
Tijo James* (1) \\ Department of Mathematics, Pavanatma College, Murickassery, Idukki - 685604, India \\ Ambat Vijayakumar \\ Department of Mathematics, Cochin University of Science and Technology, \\ Cochin - 682022, India
}

Received 4 October 2021, accepted 3 January 2022, published online 23 November 2022

\begin{abstract}
Domination game is a game played on a finite, undirected graph $G$, between two players Dominator and Staller. During the game, the players alternately choose vertices of $G$ such that each chosen vertex dominates at least one new vertex that is not dominated by previously chosen vertices. The aim of Dominator is to finish the game as early as possible while that of Staller is to delay the process as much as possible. The game domination number $\gamma_{g}(G)$ is the total number of moves in the game when Dominator starts and both players play optimally. Similarly the staller start game domination number $\gamma_{g}^{\prime}(G)$ is the total number of moves in the game when Staller starts and both players play optimally.

Here the domination game in the Mycielskian of a graph is studied. We establish bounds for the game domination number of Mycielskian of a graph in terms of its domination number and also in terms of its game domination number. The Mycielskian of a graph with small game domination number are characterised.
\end{abstract}

Keywords: Domination game, Mycielskian of a graph.

Math. Subj. Class.: 05C57, 05C69, $91 A 43$

\section{Introduction}

The Domination game was introduced in [3] by Brešar, Klavžar and Rall. A vertex dominates itself and its neighbours. In the domination game, two players, Dominator and Staller, alternate turns choosing a vertex in a finite, undirected graph $G$, and adding it to a set of

\footnotetext{
*Corresponding author.

E-mail addresses: tijojames@gmail.com (Tijo James), vambat@gmail.com (Ambat Vijayakumar)
} 
vertices $S$. The rule of the game is that whenever a player chooses a vertex to add to $S$, the vertex must dominate at least one vertex, which is not yet dominated by the vertices of $S$. The game ends when $S$ is a dominating set of the graph and the cardinality of $S$ is called the score of the game. The two players have conflicting goals - Dominator tries to minimize the final score while Staller tries to maximize it.

Two graph parameters associated with this game were introduced in [3]. Assuming both players play optimally, the game domination number $\gamma_{g}(G)$ is the score of the game on $G$ when Dominator starts ( D-game), and the Staller start game domination number $\gamma_{g}^{\prime}(G)$ is the score when Staller starts (S-game). Both parameters are studied in parallel since many results hold for both of them.

In terms of the order of the graph, Kinnersley, West and Zamani [12] conjectured that a general upper bound for $\gamma_{g}(G)$ is $\frac{3}{5}$ of its order if $G$ is an isolate free graph. Bujtás $[6,4,5]$ developed an innovative discharging-like method to attack this conjecture. The conjecture was confirmed by Henning and Kinnersley on the class of graphs with minimum degree at least two [10]. Schmidt [14] determined the largest known class of trees for which the conjecture holds. It is known that the difference between $\gamma_{g}(G)$ and $\gamma_{g}^{\prime}(G)$ is at most one, and that it can occur in both directions. The consequences of an edge removal in a graph were considered in [1] and it is proved that $\gamma_{g}(G)$ and $\gamma_{g}^{\prime}(G)$ can either increase or decrease by at most two. It is proved that in both the cases, the game domination number can either increase or decrease by at most one in the class of no-minus graphs[11]. This motivated us to study the effect of game domination number when a graph operator [13] acts on a graph.

In this paper, we study the effect of game domination number when the graph operator Mycielskian acts on a graph.

\section{Preliminaries}

A partially-dominated graph is a graph together with a declaration that some vertices are already dominated, that is they need not be dominated in the rest of the game. If $S \subseteq V(G)$, then let $G \mid S$ denote the partially dominated graph in which vertices from $S$ are already dominated. If $S=\{x\}$ we will abbreviate $G \mid\{x\}$ to $G \mid x$. If $G$ is a partially dominated graph, then $\gamma_{g}(G)$ and $\gamma_{g}^{\prime}(G)$ denote the optimal number of moves remaining in D-game and S-game, respectively (it is assumed here that D-game, respectively S-game, refers to Dominator, respectively Staller, being the first to play in the partially dominated graph $G$ ).

A vertex $u$ of a partially dominated graph $G$ is saturated if each vertex in $N[u]$ is dominated. The residual graph of $G$ is the graph obtained from $G$ by removing all saturated vertices and all edges joining dominated vertices. A vertex $u$ of a graph $G$ is a true twin of a vertex $v$ in $G$ if $N[u]=N[v]$ and a vertex $u$ of a graph $G$ is a false twin of a vertex $v$ in $G$ if $N(u)=N(v)$.

Lemma 2.1 ([12, Continuation Principle $])$. Let $G$ be a graph with $A, B \subseteq V(G)$. If $B \subseteq A$, then $\gamma_{g}(G \mid A) \leq \gamma_{g}(G \mid B)$ and $\gamma_{g}^{\prime}(G \mid A) \leq \gamma_{g}^{\prime}(G \mid B)$.

An utmost important consequence of the Continuation Principle is the following:

Theorem 2.2 ([3, 12]). If $G$ is a partially dominated graph, then $\left|\gamma_{g}(G)-\gamma_{g}^{\prime}(G)\right| \leq 1$.

A graph $G$ is a minus graph if $\gamma_{g}^{\prime}(G)=\gamma_{g}(G)-1$. A graph $G$ is a plus graph if $\gamma_{g}^{\prime}(G)=\gamma_{g}(G)+1$. A graph $G$ is an equal graph if $\gamma_{g}^{\prime}(G)=\gamma_{g}(G)$. A graph $G$ is a no-minus graph if for any subset of vertices $S, \gamma_{g}(G \mid S) \leq \gamma_{g}^{\prime}(G \mid S)$. 
Theorem 2.3 ([7]). If $G_{1} \mid S_{1}$ and $G_{2} \mid S_{2}$ are equal partially dominated no-minus graphs then the disjoint union $\left(G_{1} \cup G_{2}\right) \mid\left(S_{1} \cup S_{2}\right)$ is an equal graph and $\gamma_{g}\left(\left(G_{1} \cup G_{2}\right) \mid\left(S_{1} \cup S_{2}\right)\right)=\gamma_{g}\left(G_{1} \mid S_{1}\right)+\gamma_{g}\left(G_{2} \mid S_{2}\right)$.

Theorem 2.4 ([7]). If $G_{1}$ is an equal graph with $\gamma_{g}\left(G_{1}\right)=e$ where $e$ is an even number and $G_{2}$ is a minus graph with $\gamma_{g}\left(G_{2}\right)=o$ where $o$ is an odd number then $\gamma_{g}\left(G_{1} \cup G_{2}\right) \leq e+o$.

The Cartesian product of two graphs $G$ and $H$, denoted $G \square H$ is a graph with $V(G \square H)=V(G) \times V(H)$. The edge set of $G \square H$ consists of all pairs $\left[\left(g_{1}, h_{1}\right),\left(g_{2}, h_{2}\right)\right]$ of vertices with $g_{1}$ is adjacent to $g_{2}$ in $G$ and $h_{1}=h_{2}$ or $g_{1}=g_{2}$ and $h_{1}$ is adjacent to $h_{2}$ in $H$.

A $k$-partite graph is a graph whose vertices can be partitioned into $k$ different independent sets. A complete $k$-partite graph in which there is an edge between every pair of vertices from different independent sets. A complete multipartite graph is a complete $k$-partite graph for some $k$.

Definition 2.5. For a graph $G=(V, E)$, the Mycielskian of $\mathrm{G}$ is the graph $\mu(G)$ with vertex set $V \cup V^{\prime} \cup\{w\}$, where $V^{\prime}=\left\{u^{\prime}: u \in V\right\}$ and edge set $E \cup\left\{u v^{\prime}: u v \in\right.$ $E\} \cup\left\{v^{\prime} w: v^{\prime} \in V^{\prime}\right\}$. The vertex $v^{\prime}$ is called the twin of the vertex $v$ and vice versa. The vertex $w$ is called the root of $\mu(G)$.

\section{Bounds for the game domination number of Mycielskian of a graph}

First we obtain bounds for the game domination number of Mycielskian of a graph in terms of its domination number.

Theorem 3.1. For any graph $G, 1+\gamma(G) \leq \gamma_{g}(\mu(G)) \leq 2 \gamma(G)+1$ and $1+\gamma(G) \leq$ $\gamma_{g}^{\prime}(\mu(G)) \leq 2 \gamma(G)+2$.

Proof. It is known [3] that for any graph $G$, we have $\gamma(G) \leq \gamma_{g}(G) \leq 2 \gamma(G)-1$. So this inequality is true for $\mu(G)$ and hence $\gamma(\mu(G)) \leq \gamma_{g}(\mu(G)) \leq 2 \gamma(\mu(G))-1$. Also it is known [8] that $\gamma(\mu(G))=1+\gamma(G)$. Therefore, $1+\gamma(G) \leq \gamma_{g}(\mu(G)) \leq 2 \gamma(G)+1$.

It is known [3] that for any graph $G$, we have $\gamma(G) \leq \gamma_{g}^{\prime}(G) \leq 2 \gamma(G)$. By the above arguments we can prove that $1+\gamma(G) \leq \gamma_{g}^{\prime}(\mu(G)) \leq 2 \gamma(G)+2$.

Proposition 3.2. For any $l \geq 1$, there exists a graph $G$ with $\gamma(G)=l$ and $\gamma_{g}(\mu(G))=$ $1+l$.

Proof. Case 1: $\quad l=2 k, \quad k=1,2,3, \ldots$

Consider the graph $G$ as disjoint union of $k$ copies of $C_{4}$. Clearly $\gamma\left(C_{4}\right)=2$ and $\gamma(G)=2 k=l$. We need to prove that $\gamma_{g}(\mu(G))=1+l$. It is known [8] that $\gamma(\mu(G))=1+\gamma(G)=1+l$ and this proves $\gamma_{g}(\mu(G)) \geq 1+l$. Now we prove $\gamma_{g}(\mu(G)) \leq l+1$. For a D-game on $\mu(G)$, Dominator first plays the root vertex $w$ and the residual graph after this move is a graph with $k$ components. Each component is the graph induced by vertices of $C_{4}$ and its dominated twin vertices. Clearly game domination number of such a component is 2 . Every graph with game domination number at most 2 is no-minus.Using Theorem 2.3 we get the Staller start game domination number of the residual graph after the first move is $2 k$. Hence this D-game on $\mu(G)$ has at most $2 k+1=l+1$ moves. It is noted that Dominator's strategy may not be optimal and we get 
$\gamma_{g}(\mu(G)) \leq l+1$. This concludes that $\gamma_{g}(\mu(G))=1+l$.

Case 2: $\quad l=2 k-1, \quad k=1,2,3, \ldots$

Consider the graph $G$ as disjoint union of $k-1$ copies of $C_{4}$ and a $K_{1}$. Clearly $\gamma(G)=2(k-1)+1=2 k-1=l$ and by the same arguments above we have $\gamma_{g}(\mu(G)) \geq$ $1+l$. Now we prove $\gamma_{g}(\mu(G)) \leq l+1$. For a D-game on $\mu(G)$, Dominator first plays the root vertex $w$ and the residual graph after this move is a graph with $k$ components specifically a $K_{1}$ and the remaining $k-1$ components are the graph induced by vertices of $C_{4}$ and its dominated twin vertices. Clearly game domination number of all components other than $K_{1}$ are 2 and $\gamma_{g}\left(K_{1}\right)=1$. Every graph with game domination number at most 2 is no-minus. Using Theorem 2.3 we get the Staller start game domination number of the residual graph after the first move is $2(k-1)+1=2 k-1$. It is noted that Dominator's strategy may not be optimal and we get $\gamma_{g}(\mu(G)) \leq 1+2 k-1=1+l$. So, $\gamma_{g}(\mu(G))=1+l$.

It is proved [2] that the game domination number remains the same after attaching a true twin. Now we describe how the game domination number changes after attaching a false twin. Let $G_{u}$ be the graph obtained by attaching a false twin $u^{\prime}$ of $u$ in $G$. That is, $V\left(G_{u}\right)=V(G) \cup\left\{u^{\prime}\right\}$ and $E\left(G_{u}\right)=E(G) \cup\left\{u^{\prime} v \mid v \in N(u)\right\}$.

Lemma 3.3. For any graph $G$,

$$
\gamma_{g}(G) \leq \gamma_{g}\left(G_{u} \mid u^{\prime}\right) \text { and } \gamma_{g}^{\prime}(G) \leq \gamma_{g}^{\prime}\left(G_{u} \mid u^{\prime}\right)
$$

Proof. First we prove that $\gamma_{g}(G) \leq \gamma_{g}\left(G_{u} \mid u^{\prime}\right)$. It is enough to show that there is a strategy for Dominator on $G$ which ensures that a D-game on $G$ has at most $\gamma_{g}\left(G_{u} \mid u^{\prime}\right)$ moves. Dominator imagines a D-game on $G_{u} \mid u^{\prime}$ when a real D-game is played on $G$. The strategy of Dominator is as follows: he copies every move of Staller in the real game on $G$ to the imagined game if it is legal and responds optimally in the imagined game. He copies back his move in the imagined game to the real game on $G$ if it is legal. Every move of Staller in the real game on $G$ is a legal move in the imagined game on $G_{u} \mid u^{\prime}$ since the set of vertices that are newly dominated in $G$ with a move are also newly dominated in $G_{u} \mid u^{\prime}$. By the Continuation Principle, Dominator prefers to select $u$ in $G_{u} \mid u^{\prime}$ instead of $u^{\prime}$ in $G_{u} \mid u^{\prime}$. So every move of Dominator in the imagined game is also a legal move in the real game. Thus the real game ends when the imagined game is over. It is noted that Staller plays optimally in the real game and Dominator plays optimally in the imagined game. Hence $\gamma_{g}(G) \leq \gamma_{g}\left(G_{u} \mid u^{\prime}\right)$

The same arguments also hold for the staller start game domination number and hence $\gamma_{g}^{\prime}(G) \leq \gamma_{g}^{\prime}\left(G_{u} \mid u^{\prime}\right)$

By the Continuation Principle and Lemma 3.3 we get the following theorem.

Theorem 3.4. For any graph $G$,

$$
\gamma_{g}(G) \leq \gamma_{g}\left(G_{u}\right) \text { and } \gamma_{g}^{\prime}(G) \leq \gamma_{g}^{\prime}\left(G_{u}\right)
$$

It is known that removing a vertex from a graph can either increase its game domination number or decrease it by at most two. It is clear by Theorem 3.4 that removing a false twin from a graph never increases its game domination number. 
Theorem 3.5. For any graph $G, \gamma_{g}(G) \leq \gamma_{g}(\mu(G)) \leq 2 \gamma_{g}(G)+1$ and $\gamma_{g}^{\prime}(G) \leq$ $\gamma_{g}^{\prime}(\mu(G)) \leq 2 \gamma_{g}^{\prime}(G)+2$.

Proof. For any graph $G$, we have $\gamma_{g}(\mu(G)) \leq 2 \gamma(\mu(G))-1=2 \gamma(G)+1 \leq 2 \gamma_{g}(G)+1$. and $\gamma_{g}^{\prime}(\mu(G)) \leq 2 \gamma(\mu(G))=2 \gamma(G)+2 \leq 2 \gamma_{g}^{\prime}(G)+2$.

Now we have to prove the other part $\gamma_{g}(G) \leq \gamma_{g}(\mu(G))$. Let $(\mu(G)-w) \mid N(w)$ is obtained from $G$ by attaching a false twin (considered as dominated) to each vertex of $G$ and remove edges between two dominated vertices. So by applying Lemma 3.3 recursively we get $\gamma_{g}(G) \leq \gamma_{g}((\mu(G)-w) \mid N[w])$. The game domination number does not change by the removal of a vertex $w$ whose $N[w]$ is already dominated. Therefore $\gamma_{g}((\mu(G)-w) \mid N[w])=\gamma_{g}(\mu(G) \mid N[w])$ and by the Continuation Principle, $\gamma_{g}(\mu(G) \mid N[w]) \leq \gamma_{g}(\mu(G))$. Hence $\gamma_{g}(G) \leq \gamma_{g}(\mu(G))$.

Using similar argument above we can prove that $\gamma_{g}^{\prime}(G) \leq \gamma_{g}^{\prime}(\mu(G))$

\section{Mycielskian of a graph with small game domination number}

It is known by Theorem 3.1 that there is no graph $G$ with $\gamma_{g}(\mu(G))=1=\gamma_{g}^{\prime}(\mu(G))$.

Lemma 4.1. For any graph $G$ with at least two vertices, $\gamma_{g}(\mu(G))>2$.

Proof. Suppose that $G$ is a graph with $n \geq 2$ vertices. If $\Delta(G)=0$, then $\mu(G)$ has at least 3 components and hence $\gamma_{g}(\mu(G)) \geq \gamma(\mu(G)) \geq 3$. Thus we assume that $G$ has a non-isolated vertex say $v_{1}$. If Dominator chooses $w$ as his first move then staller plays $v_{1}^{\prime}$. If Dominator plays $v^{\prime} \in V^{\prime}$ as his first move then Staller responds by playing the vertex $w$. If Dominator plays a vertex $v \in V$ as his first move then Staller plays $u^{\prime} \in V^{\prime}$ such that $u \neq v$. In all the cases there is at least one undominated vertex after the first two moves. Thus for any graph $G$ with at least two vertices we have $\gamma_{g}(\mu(G))>2$.

Theorem 4.2. For any graph $G, \gamma_{g}(\mu(G))=2$ if and only if $G \cong K_{1}$.

Proof. It is known from Lemma 4.1 that $\gamma_{g}(\mu(G))>2$ for every graph $G$ with at least 2 vertices. Now suppose that $G \cong K_{1}$ then $\mu(G)$ is the disjoint union of a $K_{2}$ and a $K_{1}$. Thus $\gamma_{g}\left(\mu\left(K_{1}\right)\right)=2$.

Lemma 4.3. If $G$ is a non-complete graph, then $\gamma_{g}^{\prime}(\mu(G))>2$.

Proof. Suppose that $G$ is a graph having two non adjacent vertices, $u$ and $v$. Now we show that there is a strategy for Staller which ensures at least 3 moves in an $S$ game on $\mu(G)$. Suppose that Staller selects $v \in V$ as first move in $\mu(G)$. In this case Dominator cannot finish this game with his next move. If Dominator chooses $w$, the root vertex of $\mu(G)$, then the vertex $u$ is not dominated in $\mu(G)$. Again that if Dominator chooses a vertex in $V$, then the root vertex $w$ is not dominated. It is clear that the twin vertices $u^{\prime}$ of $u$ and $v^{\prime}$ of $v$ are not dominated after the first move of Staller in $\mu(G)$. So if Dominator chooses a vertex in $V^{\prime}$, then there are vertices in $\mu(G)$ which are not yet dominated. Thus there are at least 3 moves for an $S$ game on $\mu(G)$ and hence $\gamma_{g}^{\prime}(\mu(G))>2$.

Theorem 4.4. For any graph $G, \gamma_{g}^{\prime}(\mu(G))=2$ if and only if $G \cong K_{n}$.

Proof. First we show that if $G \cong K_{n}$, then $\gamma_{g}^{\prime}\left(\mu\left(K_{n}\right)\right)=2$. It is known by Theorem 3.1 that for any graph $G, \gamma_{g}^{\prime}(\mu(G)) \geq 2$. So we need to prove that $\gamma_{g}^{\prime}\left(\mu\left(K_{n}\right)\right) \leq 2$. It is enough to show that for any move of Staller in $\mu\left(K_{n}\right)$ there is a strategy for Dominator 
which ensures that there are at most two moves. Suppose that Staller selects her first move as $w$, the root vertex of $\mu\left(K_{n}\right)$. Now Dominator can finish this game by selecting a vertex $v \in V$. Clearly $w$ dominates the vertex $w$ together with all vertices in $V^{\prime}$ and the vertex $v$ dominates all vertices in $V$. So in this case there are at most two moves. Now suppose that Staller selects her first move $v^{\prime} \in V^{\prime}$. In this case Dominator can finish this game by selecting the twin vertex $v$ of $v^{\prime}$. It is clear that the vertex $v$ dominates all vertices in $V$ together with all vertices in $V^{\prime}$ except $v^{\prime}$ and the vertex $v^{\prime}$ dominates the remaining vertices $v^{\prime}$ and $w$. So this game is finished by two moves. Finally suppose that Staller selects her first move $v \in V$. Now Dominator can finish this game by selecting $w$, the root vertex of $\mu\left(K_{n}\right)$. It is clear that $v$ dominates all vertices in $V$ and $w$ dominates $w$ itself and all vertices in $V^{\prime}$. Thus we conclude that $\gamma_{g}^{\prime}\left(\mu\left(K_{n}\right)\right) \leq 2$ and hence $\gamma_{g}^{\prime}\left(\mu\left(K_{n}\right)\right)=2$.

It is known by Lemma 4.3 that if $G$ has two non adjacent vertices, then $\gamma_{g}^{\prime}(\mu(G))>2$. So $\gamma_{g}^{\prime}(\mu(G))=2$ if and only if $G \cong K_{n}$.

Lemma 4.5. If $G$ is a disconnected graph having $n$ components, then $\gamma_{g}(\mu(G)) \geq 1+n$.

Proof. Suppose that $G$ is a disconnected graph having $n$ components. Clearly $\gamma(G) \geq n$ and by theorem 3.1 we have $\gamma_{g}(\mu(G)) \geq 1+\gamma(G) \geq 1+n$.

Lemma 4.6. If $G$ is a disconnected graph having at least one edge, then $\gamma_{g}(\mu(G))>3$.

Proof. If $G$ is a disconnected graph having at least 3 components then it is known by Lemma 4.5 that $\gamma_{g}(\mu(G))>3$. So we assume that $G$ is a disconnected graph having exactly two components say $G_{1}$ and $G_{2}$ and let $e=u v$ be an edge in $G_{1}$. We prove that $\gamma_{g}(\mu(G))>3$ by showing that for any move of Dominator there is a strategy for Staller which ensures that there are at least 4 moves in a D-game on $\mu(G)$. First let us suppose that Dominator selects $w$, the root vertex of $\mu(G)$ as his first move. In this case Staller selects $u^{\prime}$ as next move. $u^{\prime}$ is a legal move for Staller since $u$ is adjacent to $v$ and hence $u^{\prime}$ dominates $v$. It is clear that no vertex in $V$ from the component of $G_{2}$ and the twin vertex $u \in V$ of $u^{\prime}$ from the component of $G_{1}$ are not dominated. So two more moves are needed to finish this game. Now suppose that Dominator selects a vertex $x^{\prime} \in V^{\prime}$ as his first move. In this case Staller selects the root vertex $w$ as her next move. It is clear that the twin vertex $x \in V$ of $x^{\prime}$ is not dominated and no vertex of $V$ from the component of $G$, which does not contain $x$ is dominated. So two more moves are needed to finish this game. Finally suppose that Dominator selects a vertex $x \in V$ of $G_{1}$. In this case Staller selects the twin vertex $x^{\prime} \in V^{\prime}$ of $x$. So the vertices and its twin vertices of $G_{2}$ are not dominated. Thus at least two more moves are needed to finish this game. Hence $\gamma_{g}(\mu(G))>3$.

Theorem 4.7. Let $G$ be a disconnected graph, then $\gamma_{g}(\mu(G))=3$ if and only if $G \cong 2 K_{1}$.

Proof. Suppose that $G$ is a disconnected graph with $\gamma_{g}(\mu(G))=3$. It is known by Lemma 4.5 that $G$ has exactly two components and by Lemma 4.6 that $G$ has no edge. In such a case $G=2 K_{1}$.

If $G \cong 2 K_{1}$ then $\mu(G) \cong K_{1,2} \cup 2 K_{1}$ and $\gamma_{g}(\mu(G))=\gamma_{g}\left(K_{1,2}\right)+\gamma_{g}\left(2 K_{1}\right)=$ $1+2=3$.

Theorem 4.8. Let $G$ be a connected graph with at least two vertices, then $\gamma_{g}(\mu(G))=3$ if and only if every vertex of $G$ lies in a connected dominating set of order 2. 
Proof. Suppose that $G$ is a connected graph with every vertex of $G$ lies in a connected dominating set of order 2. Now we show that $\gamma_{g}(\mu(G))=3$. It is known by Lemma 4.1 that $\gamma_{g}(\mu(G)) \geq 3$. Therefore it is enough to show that $\gamma_{g}(\mu(G)) \leq 3$. Now we show that there exists a strategy for Dominator which ensures at most 3 moves in a D-game on $\mu(G)$. First move of Dominator in $\mu(G)$ is the root vertex $w$. Suppose that Staller selects a vertex $u^{\prime} \in V^{\prime}$. It is known by our assumption that every vertex lies in a connected dominating set of order 2. Therefore there exist a vertex $v \in V$ which is adjacent to $u$ and $\{u, v\}$ forms a dominating set of $G$. Now Dominator can finish this game on $\mu(G)$ by selecting $v$ as next move. It is clear that $w$ dominates itself together with all vertices in $V^{\prime}$. The vertex $u^{\prime}$ dominates all vertices in $N_{G}(u)$ and the vertex $v$ dominates $u$ and all the remaining vertices in $G$. Thus $V(\mu(G))=\{w\} \cup N_{\mu(G)}\left[u^{\prime}\right] \cup N_{\mu(G)}[v]$ and this game ends by these 3 moves.

Suppose that Staller selects a vertex $u \in V$ after the first move of Dominator. By our assumption there exist a vertex $v \in V$ such that $\{u, v\}$ is a connected dominating set in $G$. Therefore it is clear that $u, v \& w$ dominates all vertices in $\mu(G)$ and hence Dominator can finish this game by selecting the vertex $v$ as his next move. So we conclude that $\gamma_{g}(\mu(G))=3$.

Conversely suppose that $G$ is a connected graph with a vertex $u \in V(G)$ such that $u$ does not belong to any connected dominating set of order 2 . It is clear that $G$ has no universal vertex (if $G$ has a universal vertex $v$, then $\{u, v\}$ is a connected dominating set of order 2). Now we show that $\gamma_{g}(\mu(G)) \neq 3$. For that it is enough to show that Staller has a strategy in a D-game on $\mu(G)$ such that the game has at least 4 moves.

Suppose that first move of Dominator is the root vertex $w$ in $\mu(G)$. Now Staller selects the vertex $u^{\prime}$ and it is clear that $u$ is not dominated in $\mu(G)$. So the game is not finished by the next move of Dominator. Since the vertex $u$ does not belong to any connected dominating set of order 2 , it is impossible to find a vertex $v$ which is adjacent to $u$ and $\{u, v\}$ is a dominating set of $G$.

Suppose that first move of Dominator is a vertex $v^{\prime} \in V^{\prime}$ in $\mu(G)$. If $v$ is adjacent to $u$, then $\{u, v\}$ is not a dominating set of $G$. Now Staller chooses $u^{\prime}$ as next move. It is clear that there exists at least one vertex say $u_{1}$ in $G$ such that $u_{1}$ is not adjacent to $u$ and $v$ in $G$. Therefore $u_{1}$ and $u_{1}^{\prime}$ are not dominated in $\mu(G)$. If Dominator chooses $u_{1}$, then $u_{1}^{\prime}$ is not dominated in $\mu(G)$ and if Dominator chooses $u_{1}^{\prime}$, then $u_{1}$ is not dominated in $\mu(G)$. If there exists a vertex $u_{2}$ which is adjacent to $u, v$ and $u_{1}$, then Dominator selects $u_{2}$ and it dominates both $u_{1}$ and $u_{1}^{\prime}$ but the twin vertex $u_{2}^{\prime}$ of $u_{2}$ is not dominated in $\mu(G)$.

If $v$ is not adjacent to $u$ in $G$, then $v$ and $u$ are not dominated in $\mu(G)$ after selecting $v^{\prime}$ by Dominator. Now Staller chooses $u^{\prime}$ as her next move. Since $u^{\prime}$ dominates itself, it is a legal move for Staller. If there exists a vertex $u_{1} \in V$ which is adjacent to both $u$ and $v$, then Dominator plays either $u_{1}$ or $u_{1}^{\prime}$. In any case the other twin vertex is not dominated and hence there are at least 4 moves. If there is no vertex which is adjacent to both $u$ and $v$, then at least two more moves are needed to finish this game. So there are at least 4 moves in this case.

Suppose that first move of Dominator is a vertex $v \in V$ in $\mu(G)$. If $v$ is adjacent to $u$ in $G$, then there exist a vertex $u_{1} \in V$ such that $u_{1}$ is not adjacent to both $u$ and $v$. Otherwise $\{u, v\}$ is a connected dominating set of order 2 in $G$ which contradicts our assumption. Clearly $v^{\prime}, u_{1}$ and $u_{1}^{\prime}$ are not dominated in $\mu(G)$ after the first move by Dominator. Now Staller chooses $u$ as her next move. Since $u$ dominates $v^{\prime}$, it is a legal move for Staller. Note that $u_{1}, u_{1}^{\prime}$ and the root vertex $w$ are not yet dominated. It is clear there is no vertex in $\mu(G)$ which is adjacent to $u_{1}, u_{1}^{\prime}$ and $w$. So at least two more moves are needed to finish 
this game and hence $\gamma_{g}(\mu(G)) \geq 4$.

If $v$ is not adjacent to $u$ in $G$, then $u, u^{\prime}$ and $v^{\prime}$ are not dominated in $\mu(G)$ after selecting $v$ by Dominator. Now Staller chooses $u$ as her next move. Since $u$ dominates itself, it is a legal move for Staller. If there exists a vertex $u_{1} \in V$ which is adjacent to both $u$ and $v$, then Dominator plays either $u_{1}$ or $u_{1}^{\prime}$. In any case the other twin vertex is not dominated and hence there are at least 4 moves. If there is no vertex which is adjacent to both $u$ and $v$, then at least two more moves are needed to finish this game. So there are at least 4 moves in this case.

Suppose that first move of Dominator is the vertex $u \in V$ in $\mu(G)$. Now Staller chooses $v \in V$, a vertex adjacent to $u$ in $\mu(G)$. Since $v$ dominates $u^{\prime}$, it is a legal move for Staller. It is clear that there exists at least one vertex say $u_{1}$ in $G$ such that $u_{1}$ is not adjacent to $u$ and $v$ in $G$. Note that $u_{1}, u_{1}^{\prime}$ and the root vertex $w$ are not yet dominated. It is clear there is no vertex in $\mu(G)$ which is adjacent to $u_{1}, u_{1}^{\prime}$ and $w$. So at least two more moves are needed to finish this game and hence $\gamma_{g}(\mu(G)) \geq 4$.

Suppose that first move of Dominator is the vertex $u^{\prime} \in V^{\prime}$ in $\mu(G)$. Now Staller chooses the root vertex $w$ as her next move. Clearly $u$ is not dominated after these two moves. By our assumption $u$ does not belong to any connected dominating set of order 2 . So at least two more moves are needed to finish this game and hence $\gamma_{g}(\mu(G)) \geq 4$.

Theorem 4.9. If $G$ is a disconnected graph, then $\gamma_{g}^{\prime}(\mu(G)) \geq 4$.

Proof. Suppose that $G$ is a disconnected graph with at least two components, say $G_{1}$ and $G_{2}$. Now we show that $\gamma_{g}^{\prime}(\mu(G)) \geq 4$. It is enough to show that there exists a strategy for Staller which ensures at least 4 moves in an S-game on $\mu(G)$. Let $V_{1}=V\left(G_{1}\right)$ and $V_{2}=V\left(G_{2}\right)$. Staller chooses her first move as $u^{\prime} \in V_{1}^{\prime}$. It is clear that the twin vertex $u$ of $u^{\prime}$ in $\mu(G)$ and all vertices in $V_{2} \cup V_{2}^{\prime}$ are not dominated. Suppose that if Dominator chooses the vertex $w$ as his move, then the vertex $u$ and all vertices in $V_{2}$ of $\mu(G)$ are not dominated. So there are at least 4 moves needed to finish this game. If Dominator selects the vertex $v^{\prime} \in V_{2}^{\prime}$ as his move, then it is clear that $u$ and $v$ are not dominated. So there are at least 4 moves needed to finish this game. If Dominator chooses a vertex $v \in V_{1}$ as his move, then it is clear that all vertices in $V_{2} \cup V_{2}^{\prime}$ are not dominated. So Staller selects a vertex in $V_{2}^{\prime}$ and its twin vertex is not yet dominated. Thus there are at least 4 moves in this game. If Dominator chooses the vertex $v \in V_{1}$ as his move, then it is clear that all vertices in $V_{2} \cup V_{2}^{\prime}$ are not dominated. So Staller selects a vertex in $V_{2}^{\prime}$ and its twin vertex is not yet dominated. Thus there are at least 4 moves in this game. If Dominator chooses a vertex $v \in V_{2}$ as his move, then Staller selects $u$ as her next move. It is clear that $v^{\prime}$, the twin vertex of $v$ in $\mu(G)$, is not dominated. Thus we conclude that $\gamma_{g}^{\prime}(\mu(G)) \geq 4$.

Lemma 4.10. If $G$ is a connected graph on $n$ vertices with $\Delta(G)<n-1$, then $\gamma_{g}^{\prime}(\mu(G)) \geq$ 4.

Proof. Let $G$ be a connected graph with $\Delta(G)<n-1$. It is clear that $G$ has at least 4 vertices. To prove that $\gamma_{g}^{\prime}(\mu(G)) \geq 4$, it is enough to show that there is a strategy for Staller which ensures at least 4 moves in an S-game on $\mu(G)$. Staller first chooses a vertex $u^{\prime} \in V^{\prime}$. Since $\Delta(G)<n-1, u$ is not adjacent to at least one vertex say $v \in V(G)$. Therefore it is clear that $u$ and $v$ are not dominated in $\mu(G)$ after the first move of Staller.

If Dominator chooses the root vertex $w$ as his move in $\mu(G)$, then Staller selects $v$ and it is clear that $u$ is not dominated by these 3 moves in $\mu(G)$. So there are at least 4 moves in this case. 
If Dominator chooses the vertex $u$, then it is clear that $v$ and its twin vertex $v^{\prime}$ are not dominated. Now Staller chooses $v$ and it is clear that $v^{\prime}$ is not dominated by these 3 moves in $\mu(G)$. So there are at least 4 moves in this case.

If Dominator chooses a vertex $u_{1} \in V$ which is adjacent to $u$, then it is clear that $u_{1}^{\prime}$ is not dominated in $\mu(G)$. Since $\Delta(G)<n-1$, there exists a vertex $u_{2}$ in $G$ such that $u_{2}$ is not adjacent to $u_{1}$. Therefore $u_{2}^{\prime}$ is not dominated in $\mu(G)$. Now Staller selects $u_{1}^{\prime}$ and it is clear that $u_{2}^{\prime}$ is not dominated by thsese 3 moves in $\mu(G)$. So there are at least 4 moves in this case.

If Dominator chooses a vertex $u_{1} \in V$ which is not adjacent to $u$, then it is clear that $u_{1}^{\prime}$ is not dominated in $\mu(G)$. Now Staller chooses $u_{1}^{\prime}$ and it is clear that $u$ is not dominated by these 3 moves in $\mu(G)$. So there are at least 4 moves in this case.

If Dominator chooses a vertex $u_{1}^{\prime} \in V^{\prime}$, then Staller chooses a vertex in $V^{\prime}$ other than $u^{\prime}$ and $u_{1}^{\prime}$. It is known that $G$ has at least 4 vertices and hence in $\mu(G)$ there is at least 1 vertex which is not yet dominated in $V^{\prime}$. So there are at least 4 moves in this case. Thus $\gamma_{g}^{\prime}(\mu(G)) \geq 4$

Theorem 4.11. For any connected graph $G, \gamma_{g}^{\prime}(\mu(G))=3$ if and only if $G \nsucceq K_{n}$ and $\Delta(G)=n-1$.

Proof. Let $G \supsetneqq K_{n}$ be a connected graph with $\Delta(G)=n-1$. It follows from Theorem 4.4 that $\gamma_{g}^{\prime}(\mu(G)) \geq 3$. So we need to prove that $\gamma_{g}^{\prime}(\mu(G)) \leq 3$. It is enough to show that there exist a strategy for Dominator which ensures at most 3 moves in an S-game on $\mu(G)$. Suppose that first move of Staller is a vertex $u \in V$ in $\mu(G)$. Note that $G \nsubseteq K_{n}$ and first move of Staller is a vertex with $d(u)<n-1$ because of Continuation Principle. Now Dominator chooses a universal vertex $v \in V$ in $\mu(G)$ (this is possible because $\Delta(G)=n-1)$. So all vertices in $V \cup V^{\prime}$ is dominated and the only vertex which is not dominated is $w$. Hence this game has at most 3 moves.

If first move of Staller is a vertex $u^{\prime} \in V^{\prime}$ in $\mu(G)$. Now Dominator chooses a universal vertex of $G$ say $v \in V$ in $\mu(G)$ (This is possible because $\Delta(G)=n-1$ ). So all vertices in $\mu(G)$ except $v^{\prime}$, the twin vertex of $v$, are dominated in $\mu(G)$. Hence this game has at most 3 moves.

If first move of Staller is the root vertex $w$ in $\mu(G)$, then Dominator can finish this game by selecting a universal vertex of $G$ say $v \in V$ in $\mu(G)$. So we conclude that $\gamma_{g}^{\prime}(\mu(G)) \leq 3$.

Conversely suppose that $\gamma_{g}^{\prime}(\mu(G))=3$ for a graph $G$. It is known by Theorem 4.4 that $G \not K_{n}$ and by Lemma 4.10 that $\Delta(G) \geq n-1$. Therefore if $\gamma_{g}^{\prime}(\mu(G))=3$, then $G \nsubseteq K_{n}$ and $\Delta(G)=n-1$.

Theorem 4.12. If $G=K_{n_{1}, n_{2}, n_{3}, \ldots, n_{p}}$ is a complete multipartite graph with $n_{i} \geq 3$ for $i=1,2, \ldots$, p then $\gamma_{g}(G)=\gamma_{g}(\mu(G))=3$.

Proof. Let $G$ be a complete multipartite graph with $V(G)=V_{1} \cup V_{2} \cup V_{3} \cdots \cup V_{p}$ and $\left|V_{i}\right|=n_{i}$ for $i=1,2, \ldots, p$. It is clear that $\gamma_{g}(G)=3$. Note that Dominator first selects a vertex from one of the sets say $V_{j}$ and then Staller responds by playing a vertex in the same set $V_{j}$ in a D-game on $G$. Since $\left|V_{j}\right| \geq 3$, there are undominated vertices after these two moves. The only undominated vertices are in $V_{j}$ and Dominator can finish the game in his next move by selecting a vertex in any of the set other than $V_{j}$.

Let $\mu(G)$ be the Mycielskian of $G=K_{n_{1}, n_{2}, n_{3}, \ldots, n_{p}}$ and $V(\mu(G))=V_{1} \cup V_{1}^{\prime} \cup V_{2} \cup$ $V_{2}^{\prime} \cup V_{3} \cup V_{3}^{\prime} \cdots \cup V_{p} \cup V_{p}^{\prime} \cup\{w\}$ where $V_{i}^{\prime}$ is the set of twin vertices of the set $V_{i}$ and $w$ is the root vertex. Dominator first selects the root vertex $w$ for a D-game on $\mu(G)$. Clearly 
all vertices in $V^{\prime}$ are dominated and Staller responds by selecting a vertex from either $V_{j}$ or $V_{j}^{\prime}$. The only undominated vertices are in $V_{j}$ after these two moves. Now Dominator can finish the game by selecting a vertex from any of the set other than $V_{j}$. In this game Dominator may not plays optimally and Staller plays optimally and hence $\gamma_{g}(\mu(G)) \leq 3$. It is known by Lemma 4.1 that $\gamma_{g}(\mu(G))>2$ for any graph $G$ with at least two vertices. So $\gamma_{g}\left(\mu\left(K_{n_{1}, n_{2}, n_{3}, \ldots, n_{p}}\right)\right)=3$.

Theorem 4.13. If $G=K_{n} \square K_{2}$ where $n \geq 3$ then $\gamma_{g}(G)=\gamma_{g}(\mu(G))=3$.

Proof. Let $G=K_{n} \square K_{2}$ where $n \geq 3$. It is clear that $\gamma_{g}(G)=3$.

Dominator first selects the root vertex $w$ for a D-game on $\mu(G)$. If Staller responds with a move say $\left(u_{1}^{\prime}, v_{1}^{\prime}\right)$, the twin vertex of $\left(u_{1}, v_{1}\right)$ in $\mu(G)$, then all vertices in the first copy of $K_{n}$ except $\left(u_{1}, v_{1}\right)$ are dominated. So Dominator can finish the game by selecting the vertex $\left(u_{1}, v_{2}\right)$ in his next move. If Staller responds with a move say $\left(u_{1}, v_{1}\right)$ after the first move of Dominator, then Dominator can finish the game by selecting a vertex in the second copy of $K_{n}$ in $\mu(G)$. A similar argument also holds when Staller selects a move in the second copy of $K_{n}$ in $\mu(G)$. Thus Dominator can finish the game in at most three moves for any move of Staller. Hence $\gamma_{g}(\mu(G)) \leq 3$. It is known by Lemma 4.1 that $\gamma_{g}(\mu(G))>2$ for any graph $G$ with at least two vertices. So $\gamma_{g}(\mu(G))=3$.

Theorem 4.14. There exist a graph $G$ with $\gamma_{g}(G)=\gamma_{g}(\mu(G))=2 k+1$ for $k=1,2,3, \ldots$.

Proof. Let $G=K_{n} \square K_{2} \cup(k-1) C_{4}$ with $n \geq 3$. It is known that $\gamma_{g}\left(C_{4}\right)=\gamma_{g}^{\prime}\left(C_{4}\right)=2$ and note that any two vertices of $C_{4}$ forms a minimum dominating set of $C_{4}$. So domination game on $C_{4}$ is independent of the players who starts the game. So for any D-game on $G$, the strategy of Staller is to play the same component of $G$ in which Dominator played. If at some stage, Dominator selects a vertex say $\left(u_{1}, v_{1}\right)$ of $K_{n} \square K_{2}$ and Staller responds this move by playing a vertex say $\left(u_{j}, v_{1}\right), j \neq 1$ of this component. Now Dominator can finish this game on this component by selecting a vertex say $\left(u_{j}, v_{2}\right)$ from $K_{n} \square K_{2}$. Now the remaining undominated vertices of $G$ are in some copies of $C_{4}$. There are exctly two moves for each of $C_{4}$ to finish the game. So this game has $3+2 k-2=2 k+1$ moves. Note that in this game Staller may not plays optimally and hence $\gamma_{g}(G) \geq 2 k+1$. It is known from Result 2.4 that if $G_{1}$ is a minus graph and $G_{2}$ is an equal graph then $\gamma_{g}\left(G_{1} \cup G_{2}\right) \leq \gamma_{g}\left(G_{1}\right)+\gamma_{g}\left(G_{2}\right)$. Thus $\gamma_{g}(G) \leq 3+2 k-2=2 k+1$.

Consider a D-game on $\mu(G)$ and Dominator first selects the root vertex $w$. Residual graph after this move is a partially dominated graph with $k$ components in which $k-1$ components are $C_{4}$ together with its twin vertices and one component is $K_{n} \square K_{2}$ together with its twin vertices. Note that twin vertices are dominated after the first move. Now Dominator can finish the game on each component by his next move after any move of Staller in that component. Suppose that if Staller chooses a vertex in the component containing $K_{n} \square K_{2}$ say either $\left(u_{1}, v_{1}\right)$ or $\left(u_{1}^{\prime}, v_{1}^{\prime}\right)$ then Dominator can finish the game by selecting the vertex $\left(u_{1}, v_{2}\right)$. Again suppose that if Staller chooses a vertex in the component containing $C_{4}$ say $u$ or $u^{\prime}$ then Dominator can finish the game by selecting a vertex adjacent to $u$ in $C_{4}$. So each component has exactly 2 moves and hence this game has $2 k+1$ total moves. Dominator may not plays optimally and Staller plays optimally in this D-game. Thus we have $\gamma_{g}(G) \leq 2 k+1$. 


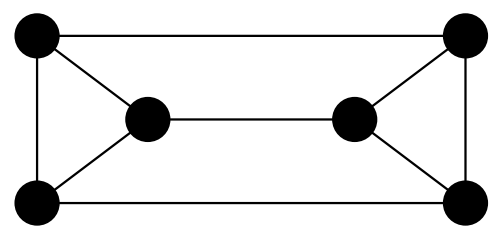

(a) $G=K_{3} \square K_{2}$

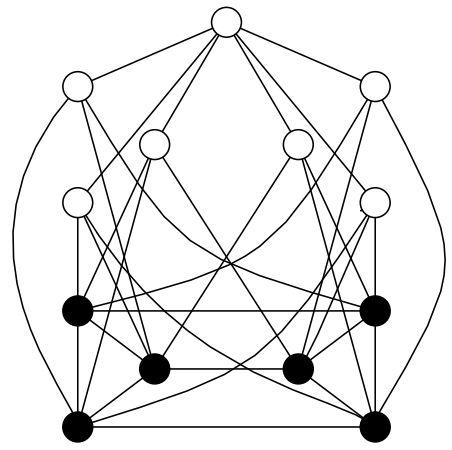

(b) $\mu\left(K_{3} \square K_{2}\right)$

Figure 1: $\gamma_{g}(G)=3=\gamma_{g}(\mu(G))$.

Note that $\gamma\left(C_{4}\right)=2$ and $\gamma\left(K_{n} \square K_{2}\right)=2$ which implies $\gamma(G)=\gamma\left(K_{n} \square K_{2} \cup\right.$ $\left.(k-1) C_{4}\right)=2+2(k-1)=2 k$. Therefore it is known from Theorem 3.1 that $1+\gamma(G) \leq \gamma_{g}(\mu(G))$. Thus $\gamma_{g}(\mu(G)) \geq 2 k+1$. Hence $\gamma_{g}(\mu(G))=2 k+1$.

\section{Concluding remarks}

We conclude the paper with the following two open problems.

Problem 5.1. Characterize graphs with $\gamma_{g}(G)=\gamma_{g}(\mu(G))$.

Problem 5.2. Does there exist a graph $G$ with $\gamma_{g}^{\prime}(G)=\gamma_{g}^{\prime}(\mu(G))$ ?

\section{ORCID iDs}

Tijo James (D) https://orcid.org/0000-0002-2486-1791

Ambat Vijayakumar (iD https://orcid.org/0000-0001-9566-3779

\section{References}

[1] B. Brešar, P. Dorbec, S. Klavžar and G. Košmrlj, Domination game: effect of edge- and vertexremoval, Discrete Math. 330 (2014), 1-10, doi:10.1016/j.disc.2014.04.015, https : / doi . org/10.1016/j.disc.2014.04.015.

[2] B. Brešar, P. Dorbec, S. Klavžar and G. Košmrlj, How long can one bluff in the domination game?, Discuss. Math., Graph Theory 37 (2017), 337-352, doi:10.7151/dmgt.1899, https: //doi.org/10.7151/dmgt.1899.

[3] B. Brešar, S. Klavžar and D. F. Rall, Domination game and an imagination strategy, SIAM J. Discrete Math. 24 (2010), 979-991, doi:10.1137/100786800, https://doi.org/10. $1137 / 100786800$.

[4] C. Bujtás, Domination game on trees without leaves at distance four, in: Proceedings of the $8^{\text {th }}$ Japanese-Hungarian Symposium on Discrete Mathematics and Its Applications (A. Frank, A. Recski, G. Wiener, eds.) June 4-7. Veszprém, Hungary, 2013 pp. 73-78, http: / /www . c. b. bme. hu/ jh2013/. 
[5] C. Bujtás, Domination game on forests, Discrete Math. 338 (2015), 2220-2228, doi:10.1016/j. disc.2015.05.022, https://doi.org/10.1016/j.disc.2015.05.022.

[6] C. Bujtás, On the game domination number of graphs with given minimum degree, Electron. J. Comb. 22 (2015), research paper p3.29, 18, doi:10.37236/4497, https: / / doi . org/ 10 . $37236 / 4497$.

[7] P. Dorbec, G. Košmrlj and G. Renault, The domination game played on unions of graphs, Discrete Math. 338 (2015), 71-79, doi:10.1016/j.disc.2014.08.024, https://doi .org/10. $1016 / j . d i s c .2014 .08 .024$.

[8] D. C. Fisher, P. A. McKenna and E. D. Boyer, Hamiltonicity, diameter, domination, packing, and biclique partitions of Mycielski's graphs, Discrete Appl. Math. 84 (1998), 93-105, doi:10.1016/s0166-218x(97)00126-1, https://doi.org/10.1016/ s0166-218x(97)00126-1.

[9] S. Foldes and P. L. Hammer, Split graphs, in: Proceedings of the Eighth Southeastern Conference on Combinatorics, Graph Theory and Computing (Louisiana State Univ., Baton Rouge, La., 1977), 1977 pp. 311-315. Congressus Numerantium, No. XIX.

[10] M. A. Henning and W. B. Kinnersley, Domination game: a proof of the 3/5-conjecture for graphs with minimum degree at least two, SIAM J. Discrete Math. 30 (2016), 20-35, doi: 10.1137/140976935, https://doi.org/10.1137/140976935.

[11] T. James, P. Dorbec and A. Vijayakumar, Further progress on the heredity of the game domination number, in: Theoretical computer science and discrete mathematics, Springer, Cham, volume 10398 of Lecture Notes in Comput. Sci., pp. 435-444, 2017, doi:10.1007/ 978-3-319-64419-6, https://doi .org/10.1007/978-3-319-64419-6.

[12] W. B. Kinnersley, D. B. West and R. Zamani, Extremal problems for game domination number, SIAM J. Discrete Math. 27 (2013), 2090-2107, doi:10.1137/120884742, https: //doi.org/10.1137/120884742.

[13] E. Prisner, Graph Dynamics, volume 338 of Pitman Research Notes in Mathematics Series, Longman, Harlow, 1995.

[14] S. Schmidt, The 3/5-conjecture for weakly $S\left(K_{1,3}\right)$-free forests, Discrete Math. 339 (2016), 2767-2774, doi:10.1016/j.disc.2016.05.017, https://doi.org/10.1016/j. disc.2016.05.017.

[15] K. Xu, X. Li and S. Klavžar, On graphs with largest possible game domination number, Discrete Math. 341 (2018), 1768-1777, doi:10.1016/j.disc.2017.10.024, https: / / doi .org/ $10.1016 / j . d i s c .2017 .10 .024$. 